\title{
Validation of a geometric compliance by the measurement of distances in the displacements space
}

\author{
Daniel Duret and Alain Sergent* \\ SYMME Laboratory, University of Savoie, BP 80439, 74944 Annecy-le-Vieux Cedex, France
}

Received: 4 March 2016 / Accepted: 9 December 2016

\begin{abstract}
The objective of the study is to show that a one-way machining simulation can be qualitatively satisfactory to validate a range of parts machined on a manufacturing with machine-tools 3 axis, even if they must meet geometric tolerances. A reflection on a method of distribution of defects is proposed. A compliance criterion based on the variance domain differences is used. Then, the main component analysis of defects is applied. The proposed model is validated by experimental analysis for which the distributions of defects are given. The results show the major character of the dimensional defect component compared to the angular components (repositioning and machining). A unidirectional approach seems so reliable when the manufactured part and its range of machining remains modest complexity.
\end{abstract}

Keywords: model and measurement 3D and 1D / spread of production / clearance space and deviation space / inertia and center of gravity / noise of measurement and dispersion

\section{Introduction}

At present, CAD tools encourage use at each stage a $3 \mathrm{D}$ approach (from the product design stage to final inspection). The 3D approach is relatively well controlled and well integrated, especially in design office [1] and metrology [2]. By cons, during manufacture, process control is complex [3]. In practice, it is mainly through the steering translation adjustment parameters (gauges tools, original program, instructions axes). The driving of setting parameters of rotation (parallelism, perpendicularity ...) is fixed by the geometry of the process (machine tools, assembly) [4]. In particular, experimental work of Bui [5] showed that:

- Quantification of angular defects requires means of complex measurement and analysis $[2,6]$.

- The dispersion of results obtained on the components of rotation is same order than the intrinsic defects obtained in production [7].

In this article, we will try to show that a $3 \mathrm{D}$ approach is not necessary in all cases, and that, a 1D approach is often sufficient and avoids a measure of heaviness and treatment. This proposal will be supported by a real experiment on machine tools.

\footnotetext{
* Corresponding author: sergent@univ-smb.fr
}

\section{Definition of a distance in space travel}

\subsection{Displacement}

The objective is to compare two characteristics of the part - a target characteristic and a realization (a machining part will be called individual).

The characteristic corresponds to the relative position of two elements of references (reference systems related to surfaces or groups of surfaces). This is a displacement of solids (reference systems of associated references):

- a translation. $T_{O, A \rightarrow B}$ (for target) and $T_{k, A \rightarrow B}$ (for the $k$ th individual);

- a rotation $R_{O, A \rightarrow B}$ (for target) and $R_{k, A \rightarrow B}$ (for the $k$ th individual).

In the book [8], it is said: it will always express the relative position of two surfaces by displacement said "significant" that superimposes geometric parameters of position of the two surfaces.

If the association of reference systems to surfaces is controlled, we can then compare the two displacements (individual and target). They are defined by the passage of the $R 1$ reference system to $R 2$ [9]. These two coordinate systems are related by the displacement $D=\left\{T_{O 1 \rightarrow O 2} ; R_{O 1 \rightarrow O 2}\right\}$, which is shown in Figure 1. 
The position of a geometric vector $\overrightarrow{A M}$ can be expressed in two reference systems (Eq. (1)):

$$
\begin{aligned}
& \overrightarrow{A M}=\left(x_{M}^{1}-x_{A}^{1}\right) \times \overrightarrow{e_{1 x}}+\left(y_{M}^{1}-y_{A}^{1}\right) \times \overrightarrow{e_{1 y}}+\left(z_{M}^{1}-z_{A}^{1}\right) \times \overrightarrow{e_{1 z}} \\
& \overrightarrow{A M}=\left(x_{M}^{2}-x_{A}^{2}\right) \times \overrightarrow{e_{2 x}}+\left(y_{M}^{2}-y_{A}^{2}\right) \times \overrightarrow{e_{2 y}}+\left(z_{M}^{2}-z_{A}^{2}\right) \times \overrightarrow{e_{2 z}} .
\end{aligned}
$$

If we called $R$ matrix (Eq. (2)):

$$
[R]=\left[\begin{array}{llll}
\overrightarrow{e_{2 x}} \times \overrightarrow{e_{1 x}} & \overrightarrow{e_{2 x}} \times \overrightarrow{e_{1 y}} & \overrightarrow{e_{2 x}} \times \overrightarrow{e_{1 z}} \\
\overrightarrow{e_{2 y}} \times \overrightarrow{e_{1 x}} & \overrightarrow{e_{2 y}} \times \overrightarrow{e_{1 y}} & \overrightarrow{e_{2 y}} \times \overrightarrow{e_{1 z}} \\
\overrightarrow{e_{2 z}} \times \overrightarrow{e_{1 x}} & \overrightarrow{e_{2 z}} \times \overrightarrow{e_{1 y}} & \overrightarrow{e_{2 z}} \times \overrightarrow{e_{1 z}}
\end{array}\right]
$$

We obtain the following equation:

$$
\left[\begin{array}{lll}
x_{M}^{2} & - & x_{A}^{2} \\
y_{M}^{2} & - & y_{A}^{2} \\
z_{M}^{2} & - & z_{A}^{2}
\end{array}\right]_{R 2}=[R] \times\left[\begin{array}{ccc}
x_{M}^{1} & -x_{A}^{1} \\
y_{M}^{1} & -y_{A}^{1} \\
z_{M}^{1} & - & z_{A}^{1}
\end{array}\right]_{R 1}
$$

Particular case where $A$ is equal to $O 1$ (Eq. (4)):

$$
\left[\begin{array}{l}
x_{M}^{2} \\
y_{M}^{2} \\
z_{M}^{2}
\end{array}\right]_{R 2}=\left[\begin{array}{l}
x_{O 1}^{2} \\
y_{O 1}^{2} \\
z_{O 1}^{2}
\end{array}\right]_{R 2}+[R] \times\left[\begin{array}{c}
x_{M}^{1} \\
y_{M}^{1} \\
z_{M}^{1}
\end{array}\right]_{R 1} .
$$

This transformation preserves the lengths and angles. It is an isometry.

\subsection{Identification of moving target (definition)}

In the case of two parallel planes, the choice of the coordinate system is quite arbitrary. There are three degrees of freedom for this choice: two translations in the plan and one orientation. The "Good mechanical meaning" restricts this choice. There are still many opportunities.

\subsubsection{Didactics example}

In this example (Fig. 2), we will confine ourselves to the position of the upper surface to the basic plane A.

The choice of $R 1$ is pretty easy on this part because we have three references (surfaces A, B and C). The choice is unique if we take the framed sides of " 40 " to fix the origin. Edge effect of $\mathrm{B}$ and $\mathrm{C}$ surfaces is very important [10]. The unique definition of a coordinate system is often implicit. This may generate an error of interpretation in the declaration of conformity.

For the particular case of target example (Fig. 3), we have the following equation:

$$
\left[\begin{array}{l}
x_{O 2 T}^{2} \\
y_{O 2 T}^{2} \\
z_{O 2 T}^{2}
\end{array}\right]_{R 2}=\left[\begin{array}{l}
x_{O 1}^{2} \\
y_{O 1}^{2} \\
z_{O 1}^{2}
\end{array}\right]_{R 2}+[R] \times\left[\begin{array}{c}
x_{O 2 T}^{1} \\
y_{O 2 T}^{1} \\
z_{O 2 T}^{1}
\end{array}\right]_{R 1} .
$$
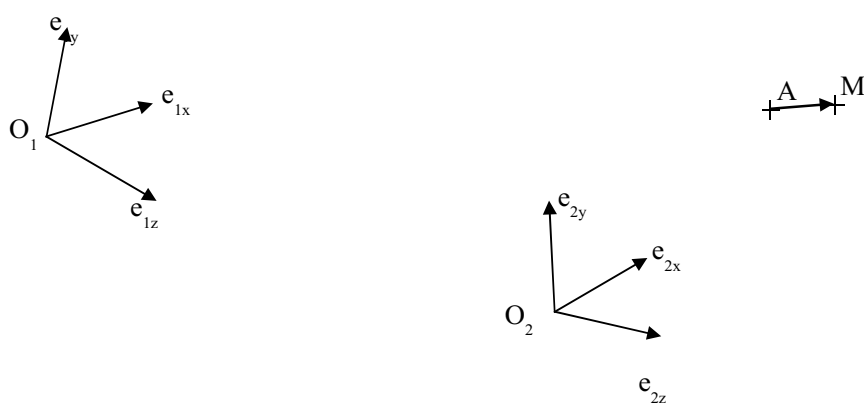

Fig. 1. Systems of coordinate.
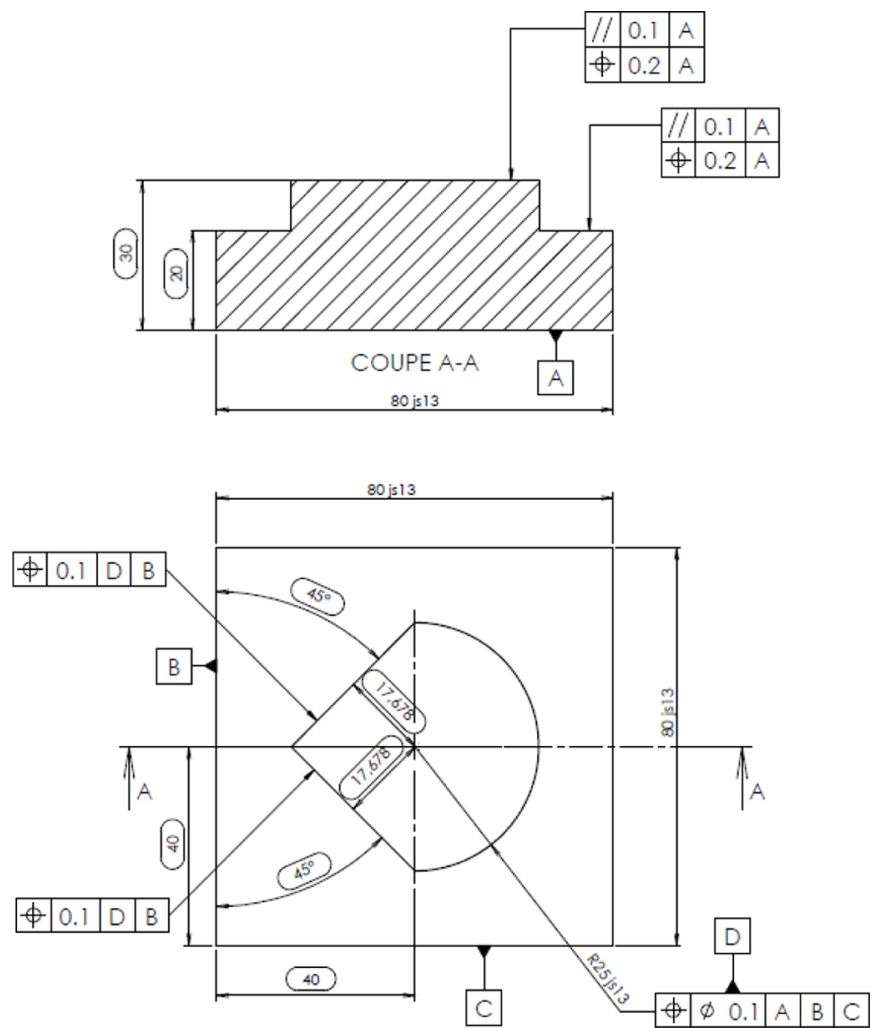

Fig. 2. Drawing of definition of the used part.

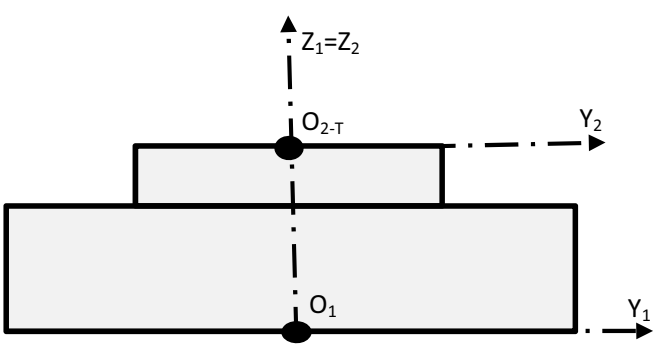

Fig. 3. Presentation of target part. 


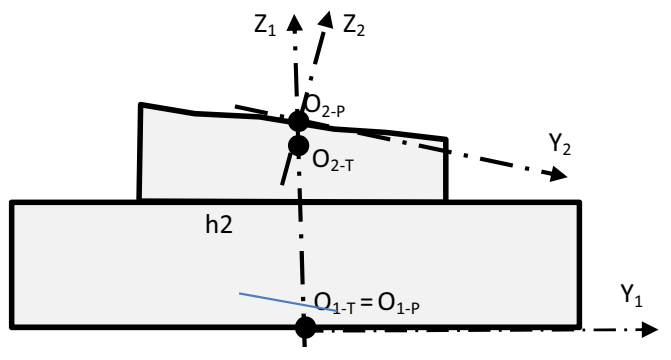

Fig. 4. Presentation of individual part.

Either numerically:

$$
\left[\begin{array}{l}
x_{O 2 T}^{2} \\
y_{O 2 T}^{2} \\
z_{O 2 T}^{2}
\end{array}\right]_{R 2}=\left[\begin{array}{l}
0 \\
0 \\
-30
\end{array}\right]_{R 2}+\left[\begin{array}{lll}
1 & & \\
& 1 & \\
& & 1
\end{array}\right] \times\left[\begin{array}{l}
0 \\
0 \\
30
\end{array}\right]_{R 1} .
$$

\subsection{Identification of displacement on an individual}

To compare the two movements (target and individual), they must be defined in the same way. This is more difficult $[7,8]$. We must first define $R 1$ on the part [using the Coordinate Measuring Machine (CMM)]. $R 1$ is associated with the part by numerical methods (least squares type).

For the particular case of individual part (Fig. 4), we have the following equation:

$$
\left[\begin{array}{l}
x_{O 2 T}^{2} \\
y_{O 2 T}^{2} \\
z_{O 2 T}^{2}
\end{array}\right]_{R 2}=\left[\begin{array}{l}
x_{O 1}^{2} \\
y_{O 1}^{2} \\
z_{O 1}^{2}
\end{array}\right]_{R 2}+[R] \times\left[\begin{array}{l}
x_{O 2 T}^{1} \\
y_{O 2 T}^{1} \\
z_{O 2 T}^{1}
\end{array}\right]_{R 1} .
$$

That to say:

$$
\begin{aligned}
{\left[\begin{array}{l}
x_{O 2 T}^{2} \\
y_{O 2 T}^{2} \\
z_{O 2 T}^{2}
\end{array}\right]_{R 2} } & \approx\left[\begin{array}{l}
u \\
v \\
-h 2
\end{array}\right]_{R 2}+\left[\begin{array}{lll}
1 & -\gamma & \beta \\
\gamma & 1 & -\alpha \\
-\beta & \alpha & 1
\end{array}\right] \times\left[\begin{array}{l}
0 \\
0 \\
30
\end{array}\right]_{R 1} \\
= & {\left[\begin{array}{l}
u+\beta .30 \\
v-\alpha .30 \\
30-h 2
\end{array}\right]_{R 2} \approx\left[\begin{array}{l}
0 \\
0 \\
-\delta h
\end{array}\right]_{R 2} . }
\end{aligned}
$$

\section{Differences between two geometric movements}

We will make the assumption that the two movements are known by their components $p$. It can therefore be represented by a point in a space of the same size $p$ (space of individuals).

\subsection{Small displacement torsor}

The distance between the two displacements (individual and target) is often modeled by a torsor of small displacements (Fig. 5). It is assumed that the two reference systems are

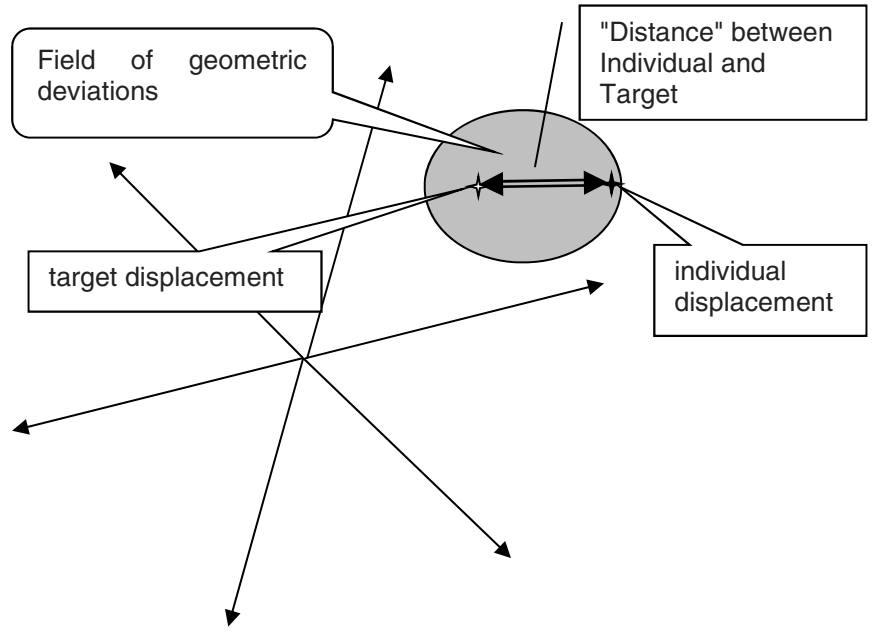

Fig. 5. Illustration of the field of geometric deviations.

aligned ( $R 1$ target and $R 1$ individual). Geometrically, we pass from the $R 2$ target to the $R 2$ individual by using a torsor of small displacement (linearization to first order).

\subsubsection{Educational example}

For example, it holds three parameters to know the difference, namely:

$-w$ : translation following axis $\mathrm{Oz}_{1}$;

$-\alpha$ : rotation about axis $\mathrm{Ox}_{1}$;

$-\beta$ : rotation about axis $\mathrm{Oy}_{1}$.

The other three parameters are identically zero. A graphic illustration in three dimensions is possible.

\section{Reflections on the distribution of the distance between two displacements}

The objective of the analysis of displacements between "target and individual" is to determine all components of displacements (individual target) and show that some are very small compared to others. This will validate a $1 \mathrm{D}$ approach is generally sufficient and much simpler implementation.

\subsection{Formatting data}

We have $n$ manufactured individuals. Each individual is characterized by components $p(p \leq 6)$.

The measurement protocol is as follows:

- the measuring coordinate system is constructed from the three planes $(\mathrm{A}-\mathrm{B}-\mathrm{C})$;

- the association criterion of least squares is used to build the associated numerical element;

- moving components machined upper plane $(h=30)$ (individuals) are calculated at the centroid of the element;

- the measurement protocol of each part is defined in Table 1;

- these data are grouped in an array $X$ : 
Table 1. Illustration of protocol measurement.

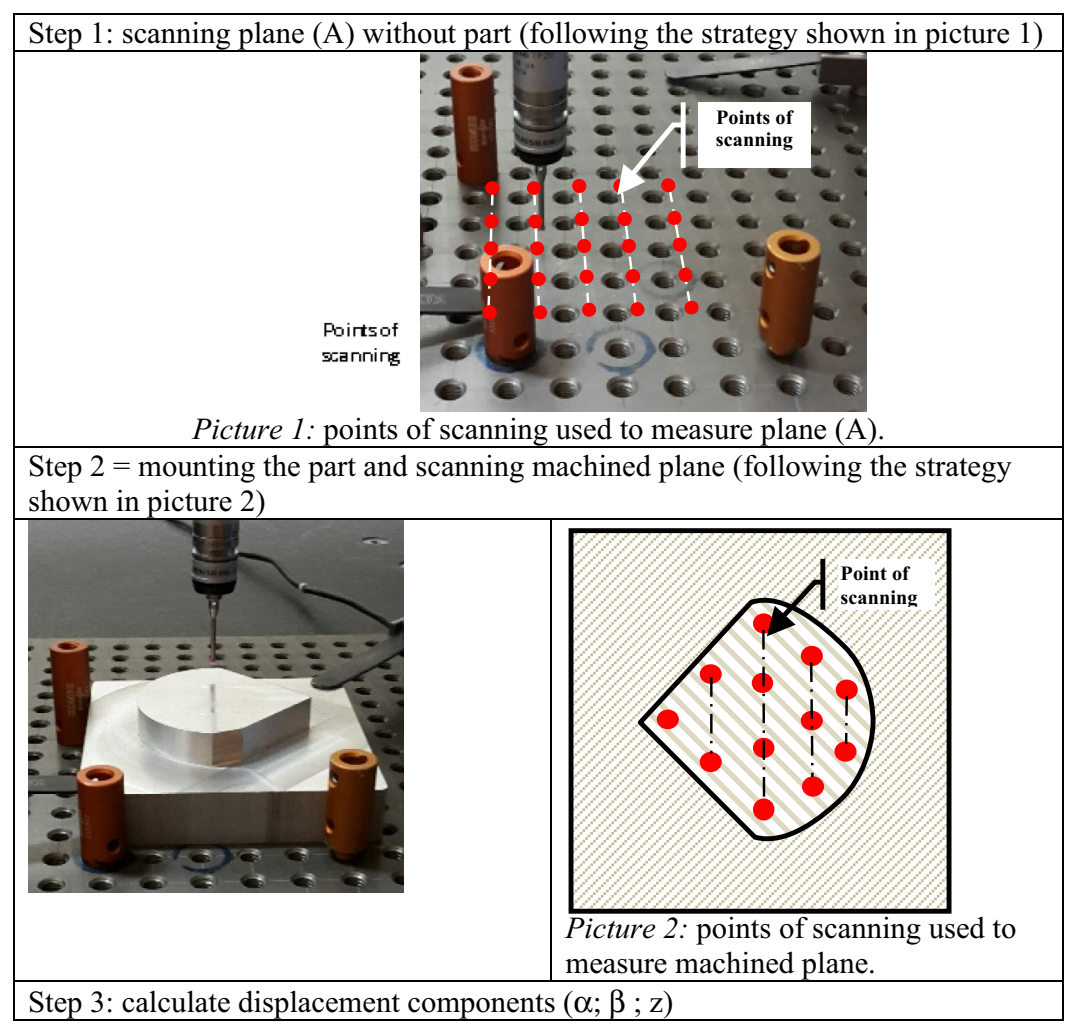

$$
[X]=\left[\begin{array}{ccccc}
x_{1}^{1} & & \cdots & & x_{1}^{p} \\
& \ddots & & & \\
\vdots & & x_{i}^{j} & & \vdots \\
x_{n}^{1} & & \cdots & & x_{n}^{p}
\end{array}\right]
$$

- An individual $\boldsymbol{e}_{\boldsymbol{i}}$ is a vector at $p$ components (it corresponds to a row in the array). A variable $x^{j}$ (column) is identified by the $n$ measured values for the same parameter.

- For our example, we get the following equation:

$$
[X]=\left[\begin{array}{ccc}
A_{1} & B_{1} & W_{1} \\
A_{i} & B_{i} & W_{i} \\
A_{n} & B_{n} & W_{n}
\end{array}\right]=\left[\begin{array}{ccc}
\alpha_{1} & \beta_{1} & W_{0}+w_{1} \\
\alpha_{i} & \beta_{i} & W_{0}+w_{i} \\
\alpha_{n} & \beta_{n} & W_{0}+w_{n}
\end{array}\right] .
$$

\subsection{The space of individuals (displacements)}

\subsubsection{Center of gravity}

Displacement (line) is defined by its $p$ coordinates. It is an element of the space of individuals. The set of $n$ individuals form a cloud of points with its center of gravity $g$ :

$$
g^{T}=\left[\bar{x}^{1} ; \cdots \bar{x}^{j} ; \cdots \bar{x}^{p}\right] .
$$

Either for the example

$$
g^{T}=[\bar{A} ; \bar{B} ; \bar{W}]=\left[\bar{\alpha} ; \bar{\beta} ; W_{0}+\bar{w}\right] .
$$

It will be assumed that every individual has the same weight. The center of gravity is an indicator of the rightness of the means of production or measure.

\subsubsection{Choice of a metric in the space of individuals}

The components of the displacement have different units (angles and lengths). The general formula of distance is of the form:

$$
d^{2}\left(e_{i} ; e_{j}\right)=\left(e_{i}-e_{j}\right)^{T} \times M \times\left(e_{i}-e_{j}\right) .
$$

The most commonly used metric is

$$
[M]=[D]_{1 / s^{2}}=\left[\begin{array}{lll}
1 / s_{1}^{2} & & \\
& 1 / s_{j}^{2} & \\
& & 1 / s_{p}^{2}
\end{array}\right],
$$

where $s$ is the experimental standard deviation.

This is equivalent to multiplying each component by $1 / s$ (this allows to be dimension less but with the drawback of $s_{i}$ close to zero). 


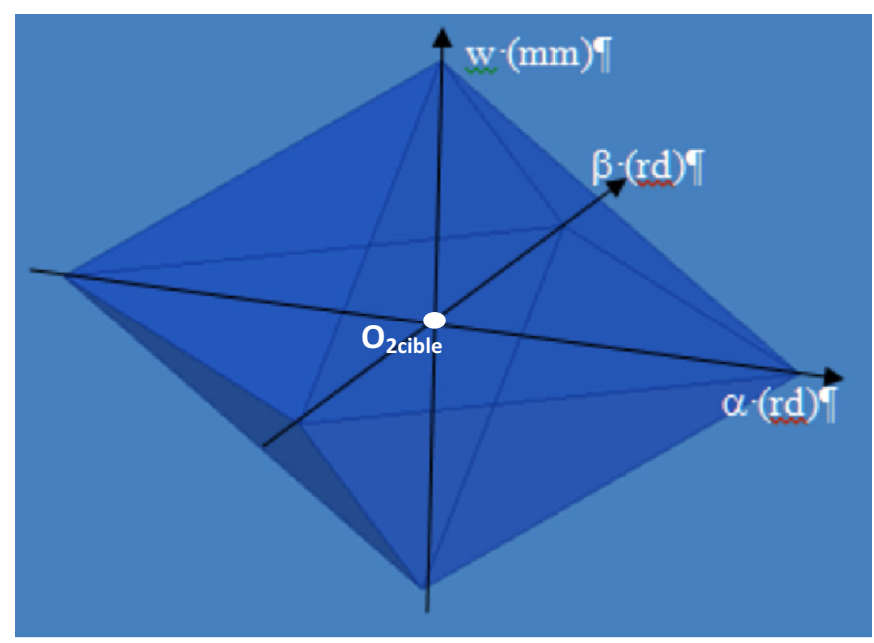

Fig. 6. The shape of the area deviations permitted.

Either for our example:

$$
[M]=[D]_{1 / s^{2}}=\left[\begin{array}{lll}
1 / s_{\alpha}^{2} & & \\
& 1 / s_{\beta}^{2} & \\
& & 1 / s_{w}^{2}
\end{array}\right]
$$

For example, the distance reduced between the 1 and 2 is equal to the following equation:

$$
\begin{aligned}
& d^{2}\left(e_{1} ; e_{2}\right)=\left(e_{1}-e_{2}\right)^{T} \times M \times\left(e_{1}-e_{2}\right), \\
& d^{2}\left(e_{1} ; e_{2}\right)=\left\|\alpha_{1}-\alpha_{2} \beta_{1}-\beta_{2} w_{1}-w_{2}\right\| \\
& \times\left\|\begin{array}{ccc}
1 / s_{\alpha}^{2} & & \\
& 1 / s_{\beta}^{2} & \\
& & 1 / s_{w}^{2}
\end{array}\right\| \times\left\|\begin{array}{c}
\alpha_{1}-\alpha_{2} \\
\beta_{1}-\beta_{2} \\
w_{1}-w_{2}
\end{array}\right\|, \\
& d^{2}\left(e_{1} ; e_{2}\right)=\frac{\left(\alpha_{1}-\alpha_{2}\right)^{2}}{s_{\alpha}^{2}}+\frac{\left(\beta_{1}-\beta_{2}\right)^{2}}{s_{\beta}^{2}}+\frac{\left(w_{1}-w_{2}\right)^{2}}{s_{w}^{2}} \text {. }
\end{aligned}
$$

\subsubsection{Inertia}

Inertia in $g$ is equal to the following equation:

$$
\begin{aligned}
I_{g} & =\sum_{i=1}^{n} p_{i}\left(e_{i}-g\right)^{T} \times D \times\left(e_{i}-g\right) \\
& =\sum_{i=1}^{n} p_{i}\left\|e_{i}-g\right\|^{2} .
\end{aligned}
$$

We consider that every individual has a weight $p_{i}$. Either the inertia in $O_{2 \text { cible }}$

$$
\begin{aligned}
I_{O 2} & =\sum_{i=1}^{n} p_{i}\left(e_{i}-e_{\text {cible }}\right)^{T} \times D \times\left(e_{i}-e_{\text {cible }}\right) \\
& =\sum_{i=1}^{n} p_{i}\left\|e_{i}-e_{\text {cible }}\right\|^{2} .
\end{aligned}
$$

The relationship of Huygens gives the following equation:

$$
I_{O 2}=I_{g}+\left\|g-e_{\text {cible }}\right\|^{2} .
$$

This scalar indicator brings together the global dispersion of the gap field and its position relative to the target. Unfortunately, it is rather difficult to interpret in the case of physical parameters of a different nature. For our example, we get with $(D=I)$ and $\left(p_{i}=1\right)$ :

$$
\begin{gathered}
I_{O 2}=\sum_{i=1}^{n}\left[\alpha_{i}-0 ; \beta_{i}-0 ; W_{0}+w_{i}-W_{0}\right] \\
\times\left[\begin{array}{lll}
1 & 0 & 0 \\
0 & 1 & 0 \\
0 & 0 & 1
\end{array}\right] \times\left[\begin{array}{l}
\alpha_{i}-0 \\
\beta_{i}-0 \\
W_{0}+w_{i}-W_{0}
\end{array}\right] \\
I_{O 2}=\sum_{i=1}^{n}\left[\alpha_{i}^{2}+\beta_{i}^{2}+w_{i}^{2}\right],
\end{gathered}
$$

with $D=M$, inertia is defined as the sum of squared distances reduced:

$$
I_{O 2}=\sum_{i=1}^{n} d^{2}\left(e_{i} ; e_{\text {cible }}\right)=\sum_{i=1}^{n} \frac{\left(\alpha_{i}\right)^{2}}{s_{\alpha}^{2}}+\frac{\left(\beta_{i}\right)^{2}}{s_{\beta}^{2}}+\frac{\left(w_{i}\right)^{2}}{s_{w}^{2}} .
$$

This definition has the advantage of use values dimensionless.

\section{Definition of compliance}

In the conventional control frame, the $p$ variables of the individual are defined by limits. Very often, these terminals are linked (not independent). For example, for the location of the upper surface, the link between the local variance $\alpha, \beta$ and $w$ can be represented by a field of permissible differences $[4,9]$.

The shape of the area deviations permitted depends strongly on the point of calculation of small displacements torsor (expressed in point $O_{2 \text { cible }}$ in Fig. 6) [11].

The series of parts will be consistent, if the domain (or ball) measured deviations is included in the permissible differences field.

For our didactics example, variables angular $\alpha$ and $\beta$ are not a problem. However, $w$ represents the projection of the vector $O_{2 \text { cible }} \vec{O}_{2 \text { individu }}$ on the axis $\mathrm{Oz}_{2 \text { cible }}$ (or $\mathrm{Oz}_{1 \text { cible }}$ for this particular case). Referring to Section 2.1, we can write:

$$
\begin{aligned}
{\left[\begin{array}{l}
x_{O_{\text {individu }}}^{2} \\
y_{O_{2 \text { individu }}}^{2} \\
z_{O_{\text {2individu }}}^{2}
\end{array}\right]_{R 2} } & {\left[\begin{array}{l}
x_{O 1}^{2} \\
y_{O 1}^{2} \\
z_{O 1}^{2}
\end{array}\right]_{R 2}+[R] } \\
& \times\left[\begin{array}{l}
x_{O_{2 \text { individu }}^{1}}^{1} \\
y_{O_{2 \text { individu }}}^{1} \\
z_{O_{2 \text { individu }}}^{1}
\end{array}\right]_{R 1}
\end{aligned}
$$


Table 2. Components of center of gravity of noise of measurement.

\begin{tabular}{lcc}
\hline \multicolumn{3}{c}{ Center of gravity $g(\mathrm{rd}, \mathrm{rd}, \mathrm{mm})$} \\
\hline $6.82 \times 10^{-4}$ & $-4.09 \times 10^{-5}$ & 0.05 \\
\hline
\end{tabular}

In our case, we go from $R 1$ to $R 2$ by a simple translation, $R$ is the identity $I$ matrix.

$$
\begin{aligned}
{\left[\begin{array}{l}
x_{O_{\text {2individu }}}^{2} \\
y_{O_{2 \text { individu }}}^{2} \\
z_{O_{2 \text { individu }}^{2}}^{2}
\end{array}\right]_{R 2} } & =\left[\begin{array}{l}
0 \\
0 \\
-W_{0}
\end{array}\right]_{R 2}+[1] \\
& \times\left[\begin{array}{l}
u \\
v \\
W_{0}+w
\end{array}\right]_{R 1} .
\end{aligned}
$$

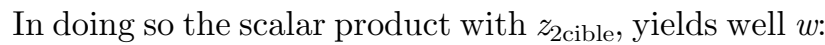

$$
\left[\begin{array}{l}
x_{O_{2 \text { individu }}}^{2} \\
y_{O_{2 \text { individu }}} \\
z_{O_{\text {individu }}}^{2}
\end{array}\right]_{R 2} \times\left[\begin{array}{l}
0 \\
0 \\
1
\end{array}\right]_{R 2}=\left[\begin{array}{l}
u \\
v \\
w
\end{array}\right]_{R 2} \times\left[\begin{array}{l}
0 \\
0 \\
1
\end{array}\right]_{R 2} .
$$

If the CMM, calculates the standard deviation $w$ following this procedure, the location of the point $O_{2 \text { individu }}$ is not fundamental.

\section{The space of variables (components of displacement)}

Each variable $x^{j}$ is a list of $n$ numeric values. It can be represented by a vector of $R^{n}$.

The "vicinity" of variables will be given by the metric defined by the diagonal matrix of weights [12].

$$
[D]=\left[\begin{array}{lll}
p_{1} & & \\
& p_{i} & \\
& & p_{n}
\end{array}\right]
$$

with for example, $p i=1 / n$, so each individual has the same weight.

The scalar product of two variables $x^{j}$ and $x^{k}$ worth:

$$
\left[x^{j}\right]^{T} \times[D] \times\left[x^{k}\right]=\sum_{i=1}^{n} p_{i} \times x_{i}^{k} \times x_{i}^{j} .
$$

If the variables are centered, the scalar product is the covariance $s_{j k}$. The magnitude of a variable is given by $\left\|x^{j}\right\|_{D}^{2}=s_{j}^{2}$. The angle $\theta_{j k}$ between two-centered variables is given by the following equation:

$$
\cos \theta_{j k}=\frac{\left\langle x^{j} \mid x^{k}\right\rangle}{\left\|x^{j}\right\| \times\left\|x^{k}\right\|}=\frac{S_{j k}}{S_{j} \times S_{k}} .
$$

The cosine is the linear correlation coefficient.
Table 3. Components of inertia of noise of measurement.

\begin{tabular}{lll}
\hline Overall inertia $I_{O 2}$ & 0.07 \\
$I_{O 2, \alpha}=1.24 \times 10^{-5}$ & $I_{O 2, \beta}=3.84 \times 10^{-8}$ & $I_{O 2, w}=0.07$ \\
Overall inertia $I_{g}$ & 0.06 & \\
\hline
\end{tabular}

\subsection{Covariance matrix}

With weights equal to $1 / n$, we obtain:

$$
[V]=[X]^{T} \times[D]_{1 / n} \times[X]-[g] \times[g]^{T} .
$$

Either for our example:

$$
[V]=\left[\begin{array}{ccc}
S_{\alpha}^{2} & \operatorname{Cov}(\alpha, \beta) & \operatorname{Cov}(w, \alpha) \\
\operatorname{Cov}(\alpha, \beta) & S_{\beta}^{2} & \operatorname{Cov}(w, \beta) \\
\operatorname{Cov}(w, \alpha) & \operatorname{Cov}(w, \beta) & S_{w}^{2}
\end{array}\right]
$$

\subsection{Linear correlation coefficients}

$$
[R]=[D]_{1 / S} \times[V] \times[D]_{1 / S}
$$

\subsection{Principal components analysis}

In the most general case, we saw that we could have six settings by individual (three in this example). The principal component analysis allows us to have a representation of individuals by their projection in a lower dimensional, that with the minimum of distortion of the distances between individuals.

In our example, you can search if the two angular axes $\alpha$ and $\beta$ can be replaced by a single axis (corresponding to that of greatest slope where assembly would result in a defect of systematic orientation). This is not the case for this experiment.

\section{Experimentation}

The experiment is carried out following 3 steps (Tab. 1): - Repeat a measurement of the same part with disassembly and reassembly on the fixture between each measurement (23 times). That allows to build a cloud of points, which shows the influence of the repositioning defect on the measuring machine.

- Measurement of a sample of parts (23 parts). The parts were products over a long period of production. A complete setting of the machine was made for each part (gauges). The series of 23 parts was machining by students. Measuring gauge tools is therefore not strictly controlled. This can highlight the sources of production defaults.

- The noise of measurement and the dispersion of recovery has been characterized by the following:

- Center of gravity of the cloud of points (accuracy) (Tab. 2).

- Inertia of the cloud of points (dispersion) (Tab. 3). 
Table 4. Components of center of gravity of spread of production.

\begin{tabular}{lcc}
\hline \multicolumn{3}{c}{ Centre of gravity $g(\mathrm{rd}, \mathrm{rd}, \mathrm{mm})$} \\
\hline $2.05 \times 10^{-4}$ & $8.95-10^{-4}$ & -0.06 \\
\hline
\end{tabular}

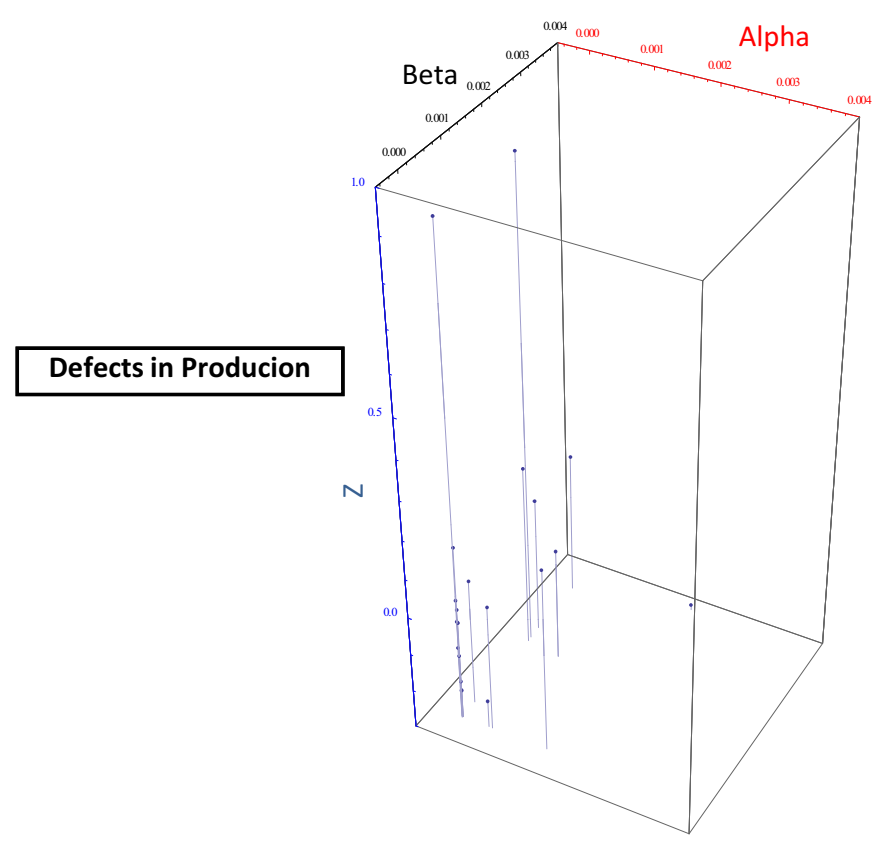

Fig. 7. Spread of the point cloud of defects in production.

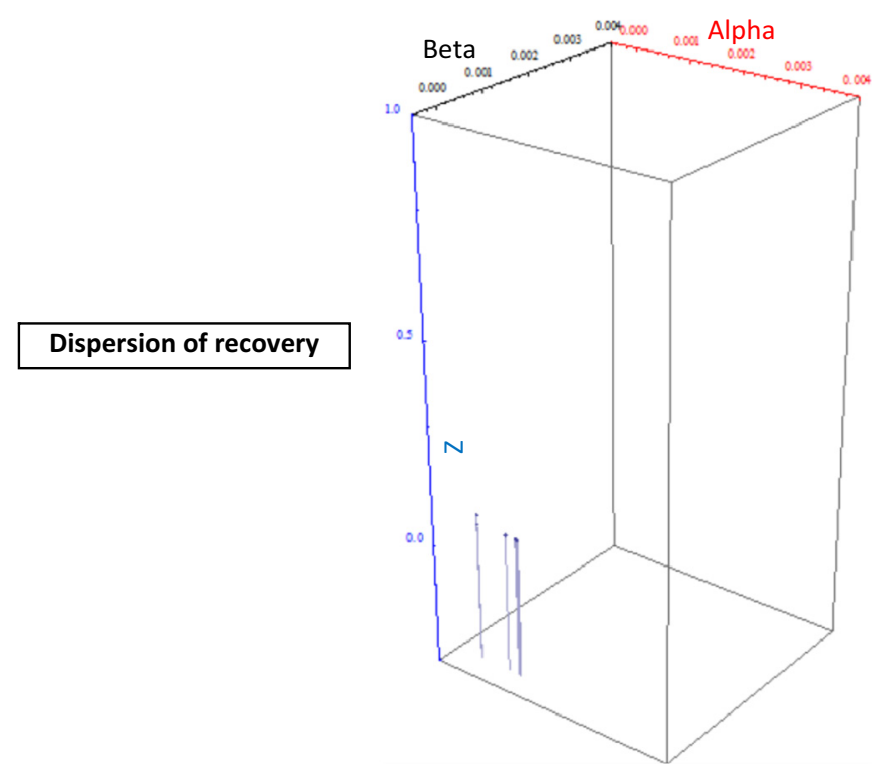

Fig. 8. Spread of the point cloud of noise of measurement and dispersion of recovery.
Table 5. Components of inertia of spread of production.

\begin{tabular}{lll}
\hline Overall inertia $I_{\mathrm{O} 2}$ & 2.18 \\
$I_{O 2, \alpha}=6.31 \times 10^{-6}$ & $I_{O 2, \beta}=6.88 \times 10^{-5}$ & $I_{O 2, w}=2.18$ \\
Overall inertia $I_{g}$ & 2.18 \\
\hline
\end{tabular}

- The spread of production has been characterized by the following:

- Centre of gravity of the cloud of points (accuracy) (Tab. 4).

- Inertia of the cloud of points (dispersion) (Tab. 5).

\section{Conclusion}

At present, production simulations favor a 3D approach [6,7]. But in a typical production system, angular instructions are rarely changed. Indeed, they are fit for mounting or machine tool (geometry). A good quality of the product fixation associated with very good geometry of machine tools take allows to control angular defects.

To obtain the geometrical conformity of a product, we need to verify that the displacement components defined previously remain below a threshold.

Otherwise, we can intervene only on some parameters of the means of production (origins programs, gauges ...).

This means that other shortcomings noted only depend on the intrinsic quality of the means of production.

In this study, we are able to quantify the components of distance that cannot be changed by the control of the means of production.

The inertia indicators defined above enable to compare and classify different means of production.

From our results, we believe that a 1D simulation is often sufficient to validate the feasibility of a range of production. Figure 7 shows that the main defect found on the batch of manufactured parts corresponds to the component along the Z-direction. The components of angular defect are much lower [13,14].

This is identical to the fixture of the part of Figure 8. Main machining defect correction can be directly accomplished on machine tools using slight modification (gauges, correctives, origin of the frame). Angular defect correction requires significant changes (change of fixture or machining).

In summary, a simulation of production validated in a 1D approach has a high probability to see actual production to meet customer requirements.

\section{References}

1. A. Desrochers, A. Rivière, A matrix approach to the representation of tolerance zones and clearances, Int. J. Adv. Manuf. Technol. 13, 630 (1997)

2. P. Bourdet, L. Mathieu, C. Lartigue, A. Ballu, The concept of the small displacement torsor in metrology, Ser. Adv. Math. Appl. Sci. 40, 110 (1996) 
3. M. Kamalinejad, F. Vignat, F. Villeneuve, Simulation of the geometrical defects of manufacturing, Int. J. Adv. Manuf. Technol. 45, 631 (2009)

4. S. Tichadou, M.K. Nejad, F. Vignat, O. Legoff, 3D manufacturing dispersions: two experimental applications, in 10th CIRP International Seminar on Computer Aided Tolerancing, Paris (2007)

5. M.H. Bui, A. Sergent, F. Villeneuve, Model of manufactured part and Monte Carlo simulation with experimental data, in 12th CIRP Conference on Computer Aided Tolerancing Manufacturing Tolerance Analysis (2010), Vol. 22

6. B.-F. Alistair, Least squares best-fit geometric elements, NPL Report DITC Affiliation (Nationale Physical Laboratory, 1989)

7. M.H. Bui, Identification et simulation des incertitudes de fabrication, PhD from the University of Grenoble, 2011

8. A. Rivière, A. Clément, M. Temmerman, Cotation Tridimensionnelle des Systèmes Mécaniques (Pyc livres, Paris, 1994)
9. A. Clément, Une science nommée technologie, Technol. Form. 108, 32 (2003)

10. A. Duret, Capabilité et pilotage d'un processus d'usinage, Technol. Form. 115, 31 (2004)

11. A. Giordano, Clearance space and deviation space. Application to three-dimensional chain of dimensions and positions, in Proc. of the 3rd CIRP Seminars on Computer-Aided Tolerancing, 1993 (1993)

12. G. Saporta, Probabilités, analyses des données et statistiques (Editions TECHNIP, 2006)

13. A. Sergent, Model and optimisation of a machining process thanks to the concept of elementary geometric minimum datum (EGMD), in 14th International CIRP, 2004 (2004)

14. M. Hien Bui, F. Villeneuve, A. Sergent, Manufacturing tolerance analysis based on the model of manufactured part and experimental data, Proc. Inst. Mech. Eng. B: J. Eng. Manuf. 5, 690 (2012)

Cite this article as: Daniel Duret, Alain Sergent, Validation of a geometric compliance by the measurement of distances in the displacements space, Int. J. Metrol. Qual. Eng. 8, 12 (2017) 